\title{
Radiation-resistant ultraviolet curable polyurethane films with CdSe-ZnS core-shell nanocrystals
}

\author{
N.R. Kulish ${ }^{1}$, V.P. Kunets*, K.Y. Narsingi ${ }^{2}$, M.O. Manasreh ${ }^{2}$, Vas. P. Kunets ${ }^{3}$ \\ ${ }^{1} V$. Lashkaryov Institute of Semiconductor Physics, NAS of Ukraine, \\ 41, prospect Nauky, 03028 Kyiv, Ukraine. \\ ${ }^{2}$ Department of Electrical Engineering, 3217 Bell Engineering Center, \\ University of Arkansas, Fayetteville, AR 72701, USA \\ ${ }^{3}$ Institute for Nanoscience and Engineering, 731 West Dickson str., \\ University of Arkansas, Fayetteville, AR 72701, USA \\ *Corresponding authore-mail:vl_kunets@yahoo.com
}

\begin{abstract}
The effect of proton fluence of different doses (up to $9 \times 10^{15}$ protons $/ \mathrm{cm}^{2}$ ) on the absorption spectra of UV-cured polyurethane films doped by $\mathrm{CdSe} / \mathrm{ZnS}$ nanocrystals has been investigated. We found that the degradation of this type of protective coating is related to the degradation of polyurethane film itself, but not the $\mathrm{CdSe} / \mathrm{ZnS}$ nanoparticles.
\end{abstract}

Keywords: proton fluence, nanocrystal, absorption spectrum, degradation.

Manuscript received 17.04.13; revised version received 18.07.13; accepted for publication 19.09.13; published online 30.09.13.

\section{Introduction}

The majority of space satellites use electricity generated by solar modules collected from a large number of photovoltaic cells in order to supply functioning onboard electronics. Triple junction solar cells, such as $\mathrm{InGaP} / \mathrm{GaAs} / \mathrm{Ge}$ [1-3], are the most efficient and preferable over the other solar cells made of $\mathrm{Si}$, InP, InGaP/GaAs, etc. [4]. Solar cells installed on spacecrafts are exposed to high energy space radiation [3] leading to degradation of their performance [1, 5]. To reduce the influence of high energy radiation (protons, neutrons, electrons, X-rays) and to extend the lifetime of the solar cells, they are protected by transparent glass with thicknesses of $0.1-0.7 \mathrm{~mm}$ [57]. To be an effective protector, the respective layers should contain light chemical elements with $Z \leq 13$, where $\mathrm{Z}$ is the nuclear charge, to protect solar cells from the harmful effects of electrons with energies $\leq 5 \mathrm{MeV}$, and heavy chemical elements for resistance to protons and X-ray radiation [6]. The first requirement is realized through polyimide and polyurethane films, while the second one is realized with the same films with embedded nanocrystals containing heavy atoms such as
$\mathrm{Cd}$, for example. It was demonstrated that resistance to 2 $\mathrm{MeV}$ proton radiation is much better for polyurethane films doped by $\mathrm{CdSe} / \mathrm{ZnSe}$ nanocrystals compared to glass [8]. Under radiation, properties of both nanocrystals and films can be changed. It is critical to understand how these changes will influence the transparency protective coatings of solar cells. In this work, we investigate the influence of radiation on the transparency of polyurethane films doped with $\mathrm{CdSe} / \mathrm{ZnS}$ nanoparticles.

\section{Experimental details}

Samples were prepared by embedding commercially available $\mathrm{CdSe} / \mathrm{ZnS}$ nanocrystals of known sizes into the polyurethane oligomer mixture. Then, this mixture was exposed to UV light. A $\mathrm{ZnS}$ shell prevents the coagulation process of $\mathrm{CdSe}$ cores and provides passivation of surface states at the boundary of the CdSe-core and $\mathrm{ZnS}$-shell.

To simulate the influence of radiation in the Van Allen belt, the films were exposed to $2-\mathrm{MeV}$ proton radiation with the fluence increments within the range $10^{13}$ to $9 \times 10^{15} \mathrm{~cm}^{-2}$. 


\section{Proton radiation influence on absorption spectra of the polyurethane films with embedded $\mathrm{CdSe} / \mathrm{ZnS}$ nanocrystals}

The effect of proton radiation on the film transparency with embedded $\mathrm{CdSe} / \mathrm{ZnS}$ nanocrystals can be demonstrated by analysis of the absorption spectra shown in Figs. 1a and 2a. The undoped polyurethane film is transparent for photons with energies $\leq 3.5 \mathrm{eV}$. The absorption spectrum of $\mathrm{CdSe} / \mathrm{ZnS}$ nanocrystals is located within the range of film transparency, and shows a characteristic spectrum due to confined states of the $\mathrm{CdSe} / \mathrm{ZnS}$ nanocrystals. The absorption spectra for $\mathrm{CdSe} / \mathrm{ZnS}$ nanoparticles, with the size close to $4.5 \mathrm{~nm}$, before and after proton irradiation (fluence $\leq 1 \times 10^{15} \mathrm{~cm}^{-2}$ ) are almost identical. For higher fluence doses, the high energy part of the spectrum shifts towards low energies. However, the spectral positions of exciton peaks are unchanged and do not depend on fluence doses (see Fig. 1a).

For films with 5-nm CdSe/ZnS nanocrystals used as a protective coating, the main disadvantage is significant absorption caused by the nanocrystals in the transparency range of the matrix. This problem can be overcome by embedding nanocrystals of smaller sizes into the films. By using the smaller nanocrystals, the absorption spectrum will exhibit a blue shift due to the increased energy separation between quantized states in the conduction and valence bands of quantum dots with smaller sizes (Fig. 2a).
For films with embedded $\mathrm{CdSe} / \mathrm{ZnS}$ nanocrystals with sizes close to $2.6 \mathrm{~nm}$, the absorption spectra are practically identical before and after exposure by proton fluence doses of $\leq 1 \times 10^{14} \mathrm{~cm}^{-2}$. An increase of proton fluence causes a low energy shift of the spectra of the polyurethane film, while the spectral position of the excitonic peaks stays the same (see Fig. 2a). Changes in the absorption spectra are a result of the following processes. The high energy protons interacting with the polyurethane film with $\mathrm{CdSe} / \mathrm{ZnS}$ nanoparticles cause: (1) breaking polymer bonds, creating a variety of new defects and establishing new polymer bonds, which lead to increased absorption at the band edge; (2) decreasing the passivation at the boundary of the CdSe core and $\mathrm{ZnS}$ shell, followed by the nucleation of large amounts of recombination centers; (3) displacement of $\mathrm{Zn}$ and $\mathrm{S}$ atoms as well as $\mathrm{Cd}$ and $\mathrm{Se}$ atoms from lattice sites of the shell and the core, respectively. These processes result in the generation of vacancies and the appearance of interstitial $\mathrm{Cd}$ and $\mathrm{S}$ atoms in the $\mathrm{ZnS}$ shell. In addition, diffusion of atoms from the core to the shell, and vice-versa, is possible under proton irradiation.

The non-stoichiometry in the CdSe core was found to be only a few percent for the samples not irradiated by protons [9]. This means that proton irradiation does not affect on the absorption spectrum of the CdSe nanocrystals but does effect on the absorption spectrum of the matrix where nanocrystals are embedded. Furthermore, the energy position of the absorption bands caused by optical transitions between the quantum
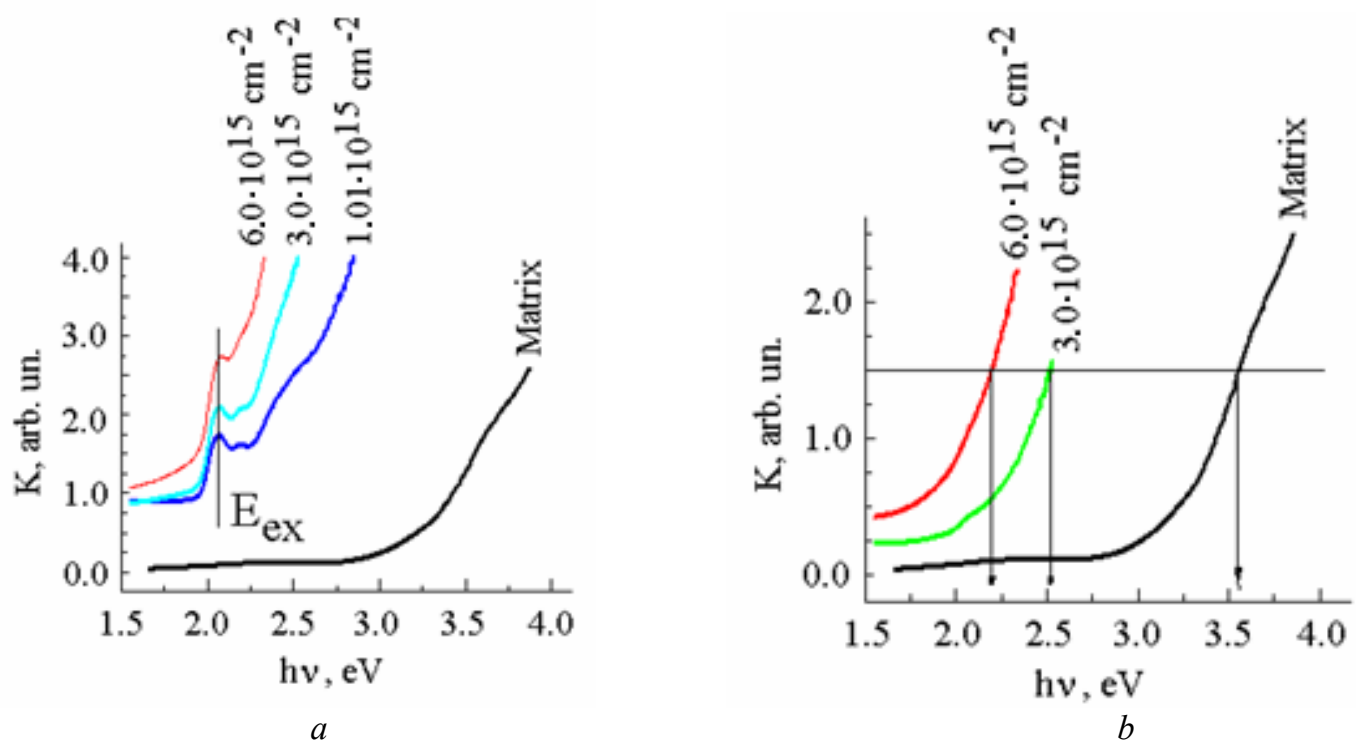

Fig. 1. Effect of proton fluence on the absorption spectra of polyurethane films with $4.5 \mathrm{~nm} \mathrm{CdSe} / \mathrm{ZnS}$ nanocrystals (a) and on absorption spectra polyurethane matrix itself (b). Reference absorption spectrum for the matrix without nanocrystals and not exposed to proton radiation is shown on both graphs. Absorption spectra for films with nanocrystals and the film without nanocrystals are shown on Figs. 1a and $1 \mathrm{~b}$, respectively, under different doses of proton radiation. $E_{\mathrm{ex}}$ corresponds to the excitonic absorption by nanocrystals. Solid line on Fig. $1 \mathrm{~b}$ represents the absorption spectrum matrix itself. Arrows indicate the energies for band edges of the matrix under different doses of proton fluence. The values of proton fluence are given for clarity. 

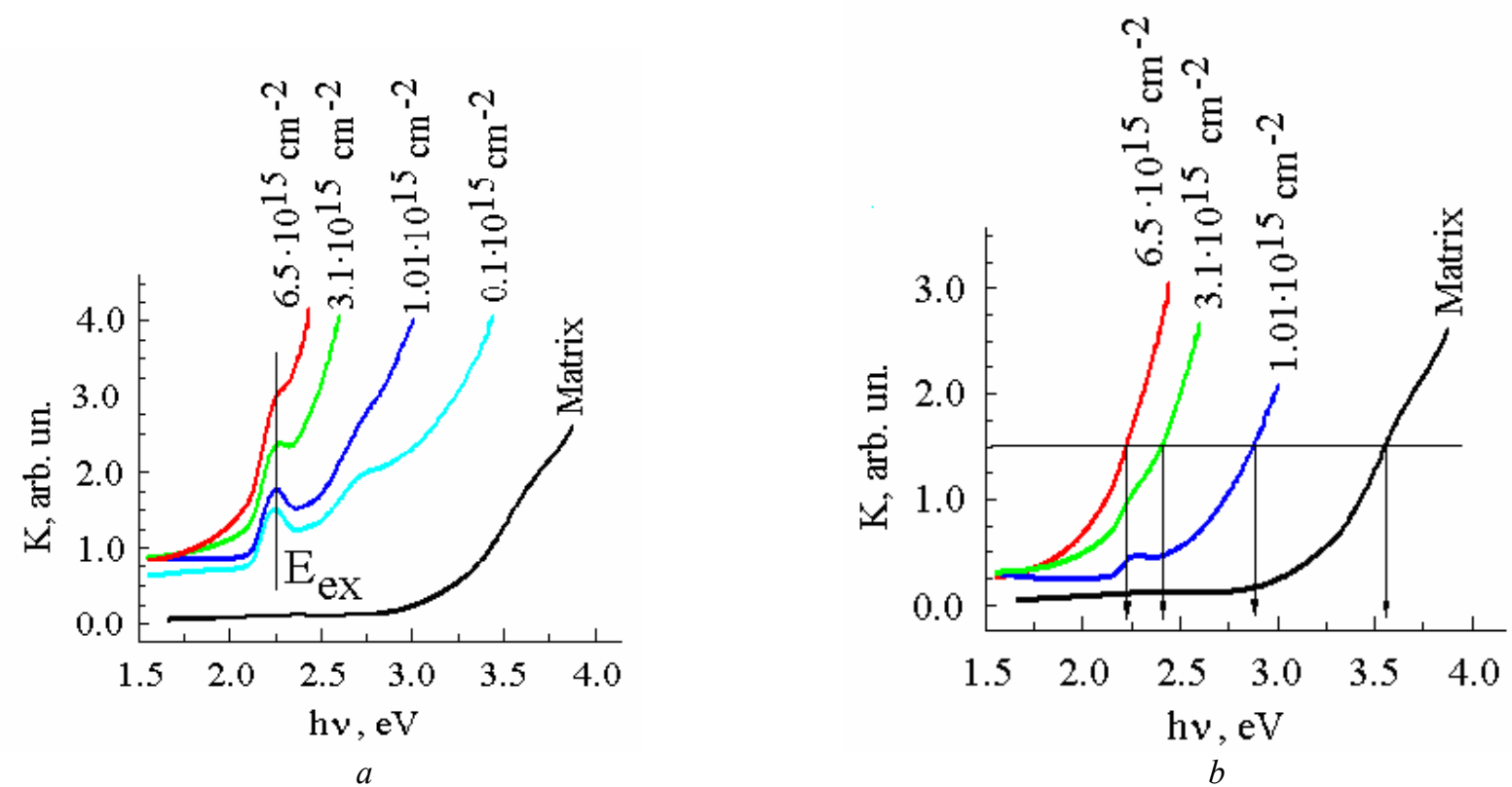

Fig. 2. Effect of proton fluence on the absorption spectra of (a) polyurethane films with $2.6 \mathrm{~nm} \mathrm{CdSe} / \mathrm{ZnS}$ nanocrystals and (b) polyurethane matrix. Reference absorption spectrum for the matrix without nanocrystals, and not exposed to proton radiation is shown on both graphs. Absorption spectra for films with nanocrystals and the film without nanocrystals are shown on Figs. 2a and $2 b$, respectively, under different doses of proton radiation. $E_{\mathrm{ex}}$ corresponds to the excitonic absorption by nanocrystals. Solid line on Fig. $2 b$ represents the absorption coefficient for the reference matrix. Arrows indicate the energies for band edges of the matrix under different doses of proton fluence. The values of proton fluence are given for clarity.

confined states of the nanocrystals does not depend on the value of the proton fluence (see Fig. 1a and 2a). However, changes in the shape of the absorption spectra are present mainly due to an increase of defect concentration in the matrix with an increase of the proton fluence.

In order to estimate influence of the proton irradiation on the energy position of the absorption spectrum inherent to the matrix itself, one could subtract the absorption spectrum of the matrix with nanocrystals non-irradiated by protons from the absorption spectrum of the proton irradiated one (Figs. $1 b$ and $2 b$ ). We note here that the results of such a procedure shows the energy position of the absorption spectrum for the matrix with the 4.5-nm nanocrystals (exposed to proton fluence of $1.01 \times 10^{15} \mathrm{~cm}^{-2}$ ) fits well to the absorption spectrum of the non-irradiated matrix. We observed the same result for the matrix with 2.6-nm nanocrystals exposed to the proton fluence $1.0 \times 10^{14} \mathrm{~cm}^{-2}$. Figs. $1 \mathrm{~b}$ and $2 \mathrm{~b}$ demonstrate the effect of proton fluence on the transparency of the protective coating made of the polyurethane film doped by $\mathrm{CdSe} / \mathrm{ZnS}$ nanocrystals.

One can estimate the effect of proton irradiation on protective coatings made of polyurethane films by comparing the measured absorption spectra to the AM0 standard [10], as it is demonstrated in Fig. 3. It is obvious that proton irradation results in the shift of the absorption band edge of coating and in the decrease of the solar power incoming to the solar cell. In particular, for the fluence close to $6.5 \times 10^{15} \mathrm{~cm}^{-2}$ more than half of the incident solar light cannot reach the solar cell.

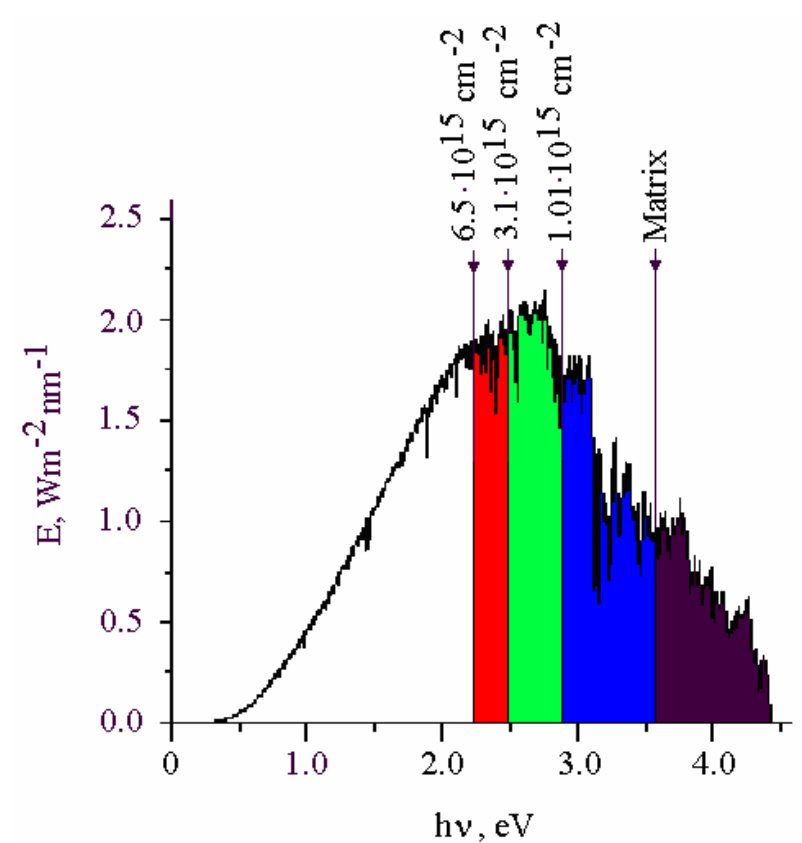

Fig. 3. Demonstration of the effect of proton fluence on the use of solar energy for electrical power generation. Arrows represent the spectral positions of band edges of the matrix for different doses of proton fluence. Similarly, the band edge for the reference matrix without nanocrystals and not exposed to protons is shown. 


\section{Conclusions}

Optical degradation of polyurethane films with embedded $\mathrm{CdSe} / \mathrm{ZnS}$ nanocrystals is a result of degradation of the polyurethane film itself, but is not affected by the change in the properties of the nanocrystals. For the proton fluence $1 \times 10^{14} \mathrm{~cm}^{-2}$, these films show a significant reduction in the level of protection for solar cells.

\section{References}

1. S.R. Messenger, E.A. Burke, R.J. Walters, J.H. Warner, G.P. Summers, T.L. Morton, Effect of Omnidirectional Proton Irradiation On Shielded Solar Cells // IEEE Trans. Nuclear Science, 53 (6), p. 3771-3778 (2006).

2. N.S. Fatemi, H.E. Pollard, H.Q. Hou, P.R. Sharps, Solar array trades between very high-efficiency multi-junction and $\mathrm{Si}$ space solar cells // 28-th IEEE Photovoltaic Specialists Conference, Anchorage, Alaska, 2000, P. 1083-1086.

3. R.R. King, C.M. Fetzer, D.C. Law, K.M. Edmondson, H. Yoon, G.S. Kinsey, D.D. Krut, J.H. Ermer, P. Hebert, B.T. Cavicchi, N.H. Karam, Advanced III-V multi-junction cells for space // 4-th World Conference on Photovoltaic Energy Conversion, Waikoloa, Hawaii, May 7-12, 2006.
4. Masafumi Yamaguchi, Radiation-resistant solar cells for space use // Solar Energy Materials \& Solar Cells, 68, p. 31-53 (2001).

5. T.V. Torchynska and G. Polupan, High efficiency solar cells for space applications // Superficies y Vacio 17(3), p. 21-25 (2004).

6. V.A. Letin, L.S. Gatsenko, T.A. Ageeva, V.F. Surkova, Protective coatings for solar spacecraft with a great resource // Nuclear Power: Technological Progress and the Economy, No 2425, S. 3-13 (2008-2009).

7. L.S. Novikov, Radiation Exposure Spacecraft Materials. Moscow, University Book, 2010.

8. K.Y. Narsingi, M.O. Manasreh, B.D. Weaver, Proton Irradiation Effect on CdSe-ZnS Core-Shell Nanocrystals Embedded in Ultra Violet Curable Resin // 2007 IEEE Region 5 Technical Conference, April 20-21, 2007, Fayetteville, AR.

9. S.J. Rosenthal, J. McBride, S.J. Pennycook, L.C. Feldman, Synthesis, surface studies, composition and structural characterization of CdSe, core/shell and biologically active nanocrystals // Surf. Sci. Reports 62, p. 111-157 (2007).

10. ASTM G173 - 03el Standard Tables for Reference Solar Spectral Irradiances: Direct Normal and Hemispherical on $37^{\circ}$ Tilted Surface Subcommittee: G03.09 Book of Standards Volume 14.04 DOI: 10.1520/G0173-03E01. 